\title{
Modeling Soil Electrical Conductivity Under Impulse Discharge for Electrical Ground System
}

\author{
Fawwaz Eniola Fajingbesi, Nur Shahida Midia, Elsheikh M. A. Elsheikh, Siti Hajar Yusoff
}

\begin{abstract}
Soil electrical conductivity play an essential role in proper grounding system design for dissipation of static and dynamic electric charge. However, soil properties such as $\mathrm{pH}$, moisture and nutrients causes' non-linear variation in the soil electrical conductivity. This non-linear soil impedance phenomena directly affect electrical modeling of soil characteristics for efficient grounding system design. In this paper, the electrical dependency of soil properties on overall impedance have been investigated and used to formulate a soil impedance electrical circuit equation model through simulation and empirical methods. Electrical conductivity for samples of $30 \mathrm{~kg}$ of soil with varying properties within at a moisture range of $14 \%-26 \%$ where injected with impulse discharge and the corresponding plot where fitted with electrical circuit simulation until a proper fit was achieved. The result shows that soil electrical impedance can be modeled with a complex circuit having both capacitive, resistive and inductive elements.
\end{abstract}

Keywords - Equivalent circuit model, Grounding system, Impulse discharge, Soil conductivity, Soil Ionization.

\section{Introduction}

Electrical grounding systems are essential necessity for all electrically connected appliances. The earth consisting of various water and soil bodies serves as the universal grounding outlet where excess electric charges are deposited. Outside man-made electric charges from their day -day activities that require grounding terminals, the atmospheric electric charges are grounded through numerous phenomena mostly lightning strike to maintain a net charge equilibrium. Since time immemorial, the empirical behavior of grounding systems were predicted either using electromagnetic computations or electrically equivalent circuit models which usually was assumed to fail in accurate prediction under transient conditions because they usually lack to fully account for current rate-of-rise and soil ionization to breakdown phenomena [1,2]. Generally, under the equivalent circuit models, the grounding impedance is represented either as a distributed circuit or as

Fawwaz Eniola Fajingbesi

Department of Physics Electronics and Earth Science

Fountain University Osogbo Nigeria

${ }^{1}$ Fawwaz Eniola Fajingbesi

${ }^{2}$ Nur Shahida Midia

${ }^{3}$ Elsheikh M. A. Elsheikh

${ }^{4}$ Siti Hajar Yusoff

${ }^{1,2,3,4}$ Department of Electrical and Computer engineering

International Islamic University Malaysia

(F.E. Fajingbesi),

(N. S. Midi),

(E. M. A. Elsheikh)

(S. H. Yusoff) a lumped circuit whose elements are combined into strata to give only a single resistance $R$, inductance $L$, and capacitance $C$ respectively as required. Circuit models are usually simple, fast, and easily recreated using standard simulation programs whose results can also be directly incorporated as a part of an overall power system real time and transient analysis.

A drawback to equivalent circuit modeling of grounding systems is its failure to usually account for the soil intrinsic properties like $\mathrm{pH}$, available moisture and nutrients which are responsible for the non-linear soil impedance phenomena that eventually dictate the rate of soil ionization and impedance breakdown but rather focused on the external impulse stimuli initiating the events such as the rate of change of injected current, intensity and leading voltage $[3,4]$.

As it is necessary to ascertain the dependency of this soil intrinsic properties on equivalent circuit models so as to better design grounding devices, the transient ground impedance of the soil in an equivalent circuit model under the injection of impulse current discharge should be able to account for the time dependent dynamic changes and show the complex non-linear characteristics observe during empirical soil electrical characterization.

Hence, this article build upon previous work by the authors [2,3] on empirical current distribution in soil and extends the study further to include an equivalent electrical model for the empirically observed soil phenomena under impulse discharge. Thus, the improved equivalent circuit model of the complex grounding impedance obtained can be directly applied or connected to power system equipment in the same simulation platform for accurately capturing the effects and benefits of grounding systems on the transient performance of electrically connected appliances. Subsequently, a better protection and insulation characteristics can be designed and optimized for various locations.

\section{Developing the Equivalent Soil Electrical Circuit Model}

Indeed, in the realm of charge transport; a material would either be a conductor, or an insulator or a semiconductor in general. Further classifications of material such as superconductors are derivatives of this initial three states. Despite this three explicit classification, a material under certain condition can be altered around the classes through external influence acting on them. A simple conductive material can have a lump electrical model defined by ohms law or a complicated electrical model defined by the Maxwell's equation. In reality, Ohms law is a simplification of the Maxwell's provided the material obeys 
Proc. of the Eighth International Conference On Advances in Computing, Electronics and Electrical Technology - CEET 2018 Copyright $\odot$ Institute of Research Engineers and Doctors. All rights reserved.

ISBN: 978-1-63248-144-3 doi: 10.15224/ 978-1-63248-144-3-29

a liner resistivity to the flow of electric charge. However, a material would only obey ohms law provided that its break point isn't exceeded just like a spring would deform after its elastic limit is exceeded. Hence the resistance $R$ of a linear material is defined as the ratio of the maximum voltage $V_{\mathrm{p}}$ to the maximum current $I_{\mathrm{p}}$

$$
R=\frac{V_{p}}{I_{p}}
$$

Subsequently, for materials with nonlinear characters, their resistance to the flow of electric charge is computed as impedance $Z$ and can be modeled with the effective resistance of the basic circuit elements (capacitance, inductance and resistance $R-L-C$ ) respectively. When a material electrical properties is properly computed as a function of time, then the transient plot of the mathematical simulation would fit the empirically obtained result with little to no variation.

\section{A. Empirical Soil Impedance Variation}

According to the practical structure of soil, it is equivalent to a porous material with different pore sizes randomly distributed as shown in the Fig. 1 below [5]. A proper modeling of this porous structure and its solid skeleton provides solution to the conductivity and breakdown characteristics of soil down to its ionization phenomena as this accounts for the microscopic layering of materials present in the soil level rather than the lump macroscopic model generally adopted as recommended by the CIGRE and the IEC 62305-1 standards [1]

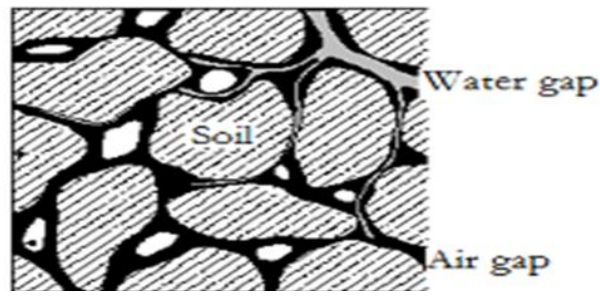

Figure 1. Soil structure

When such soil model are used, either single layer soil model or the multilayered soil model, a distinct resistivity is assigned as a lump value for each layer which failed to fully account for the breakdown and ionization phenomena recorded in the empirical results of the work by [2,3]. The effect of a variation in the soil intrinsic properties and how they affect the nonlinear soil impedance and electrical conductivity $E C$ phenomena is then presented below in Table 1. The soil EC can been seen from the presented statistics that intrinsic properties do really affect the electrical behavior of any selected soil material.

TABLE I. SOIL EC VARIATION DUE To INTRINSIC PROPERTIES

\begin{tabular}{|c|c|c|c|c|c|c|}
\hline \multicolumn{7}{|c|}{ Selected Sample Constituents } \\
\hline $\begin{array}{l}\text { Soil } \\
(\mathrm{kg})\end{array}$ & $\begin{array}{l}\mathrm{H}_{2} \mathrm{O} \\
(m L)\end{array}$ & $\begin{array}{l}\text { NPK 11- } \\
11-11(\mathrm{~g})\end{array}$ & $\begin{array}{c}N P K 4- \\
16-16(\mathrm{~g})\end{array}$ & $\begin{array}{c}\mathrm{Ca}(\mathrm{OH})_{2} \\
(\mathrm{~g})\end{array}$ & $\begin{array}{c}\text { Moisture } \\
\text { [\%] }\end{array}$ & $\begin{array}{c}\text { Conductivity } \\
\text { [mS/m] }\end{array}$ \\
\hline 30 & 0 & 0 & 0 & 0 & 14.07 & 10.64 \\
\hline 30 & 3000 & 0 & 0 & 0 & 23.16 & 20.42 \\
\hline 30 & 3300 & 100 & 0 & 0 & 23.98 & 29.31 \\
\hline
\end{tabular}

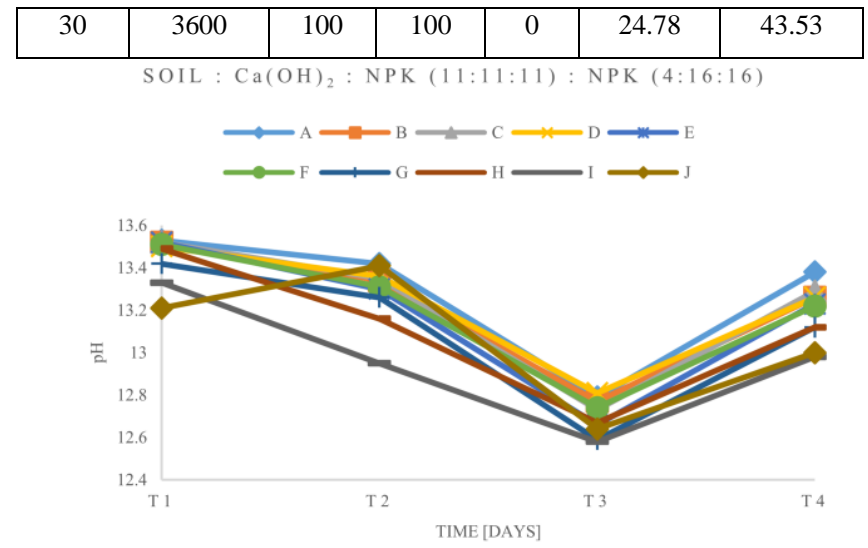

\begin{tabular}{|l|l|l|l|l|l|l|}
\hline 30 & 3900 & 100 & 100 & 100 & 25.57 & 57.89 \\
\hline
\end{tabular}

Figure 2. Time dependent Soil $\mathrm{pH}$ Variation

Furthermore, decay and instability of soil $\mathrm{pH}$ with time is also seen as shown in Fig. 2 when the soil sample are not even subjected to any discharge current. This further validate that soil properties on their own are not fixed but dynamic material subjected to a constant pedogenic process hence an accurate model for soil should be one such that is computed as a function of time. Therefore, the impedance $Z$ variation should be defined in the form of a ratio between the instantaneous maximum voltage $V_{\mathrm{p}}(\mathrm{t})$ to the instantaneous maximum inducible current $I_{\mathrm{p}}(\mathrm{t})$

$$
Z(t)=\frac{V_{p}(t)}{I_{p}(t)}
$$

By implementing equation (2), to the soil sample of Table 1 under standard lightning impulse discharge $(1.2 / 50 \mu \mathrm{s})$, a nonlinear variation is observed between the injected voltage and the induced current as shown in Fig. 3 below.

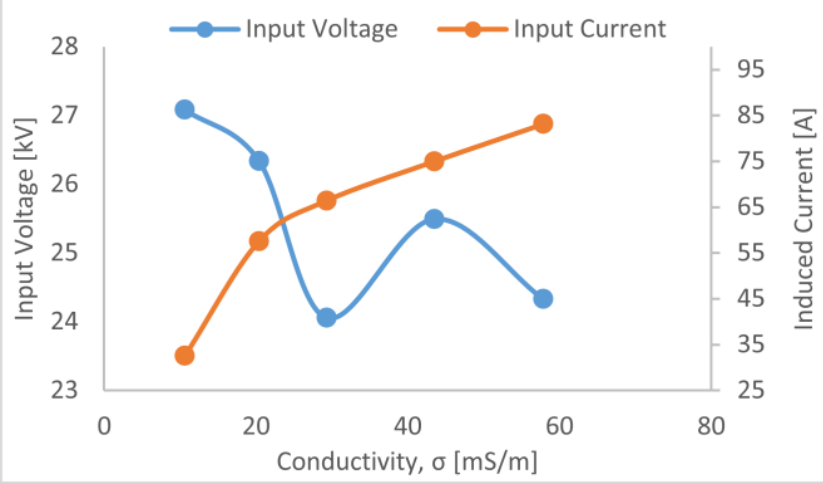

Figure 3. Instantaneous Injected Voltage and Induced current for soil with variation in conductivity

\section{B. Equivalent Soil Circuit Model}

Using NI Multisim 14, two equivalent circuit model was created for the impulse discharge process on soil using basic elements $(R-L-C)$ as building blocks for a lumped model. The initial values and locations for each circuit element illustrated in Fig. 4 were selected and swept across the range 
specified in the IEEE 1410 standard and the CIGRE recommendations[1].

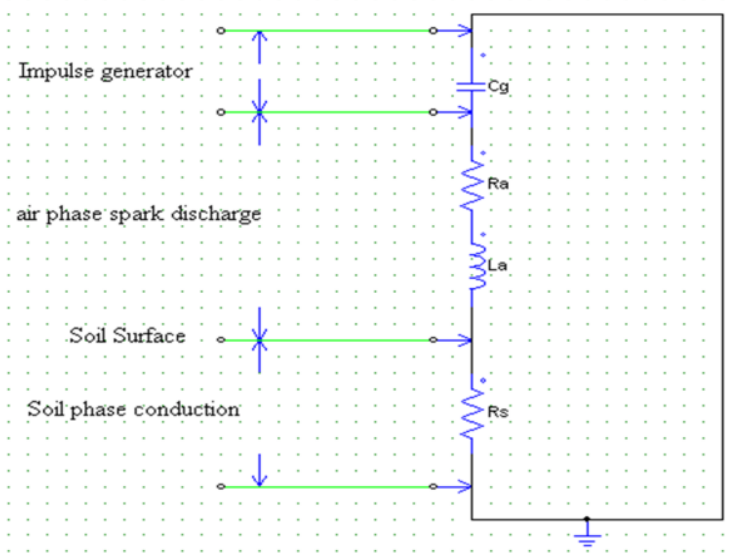

Figure 4. Conventional Soil Equivalent Circuit Model Design

The transient simulation plot obtained was then compared with the empirical result in [2,3] until a proper fit were obtained. The necessary adjustment done to obtain a proper fit in transient plot provided additional component leading to the improved equivalent electric circuit model in Fig. 5. Hence, the grounding impedance obtained at any instance from the linear circuit of Fig. 5 offer a better accuracy and improvement to the work of [6]. Hence the complex time varying equivalent impedance $Z_{0}(t)$ is calculated from the equation (3)

$$
Z_{o}(t)=\frac{V(t)}{C \frac{d V(t)}{d t}+\frac{V(t)}{R(t)}\left(1-e^{-R(t) / L(t)}\right)}
$$

When the electric field exceed the critical value defined by the capacitive, inductive and resistive elements, soil ionization leading to breakdown is observed and thus the equation for the further nonlinearity in the grounding resistance as influenced by the soil ionization phenomena is obtained by equation (4) as described in the work of [6].

$$
Z(t)=Z_{o}(t) e^{\left(-\frac{E_{n}-N}{Q_{o}}\right)}
$$

Where:

$Q_{o}$ is the thermal soil constant $(\mathrm{J} / \mathrm{m}) ; E_{n}$ is the integral of power dissipated during the ionization $(\mathrm{J} / \mathrm{m})$ and $N$ is the integral of power dissipated as heat $(\mathrm{J} / \mathrm{m}) . V, C, R$ and $L$ are voltage, capacitance, resistance and inductance respectively.

\section{Analysis}

From the model in Fig. 4, a total of 20 test were conducted each for the soil samples whose conductivity are shown in Table 1. Their simulated result all follow similar pattern as shown in Fig. 6 with slight variation in their peak values. This behavior on the plot shows that both injected and induced voltage and current both follow the same double-exponential waveform of standard lightning impulse discharge current. This signifies that a soil under impulse discharge can not be

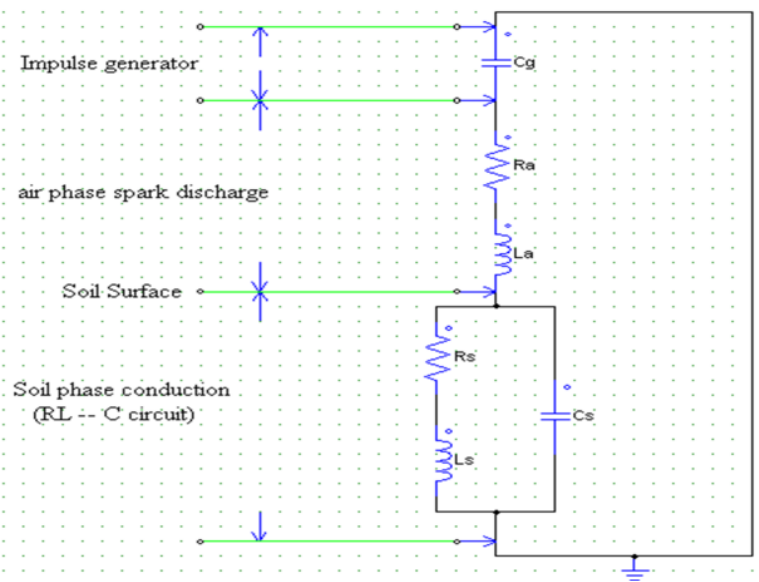

Figure 5. Improved Soil Equivalent Circuit Model Design

properly modelled using only a resistive element. Similarly, from the improved model in Fig. 5, a total of 20 test was carried out each just as in the initial model. Their simulated results were also in the form of the plot shown in Fig. 7 which corresponds to the empiracal result of $[2,3]$. The soil model here defined by the equations (3) shows that in the equivalent model for a soil under imulse discharge; inductive, resistive and capacitive elements are always present. The current in this model is observed to instatntaneously rise to its peak value then fall just only to rise again with another minor peaks. This second peak are the manifestation of material breakdown and soil ionization

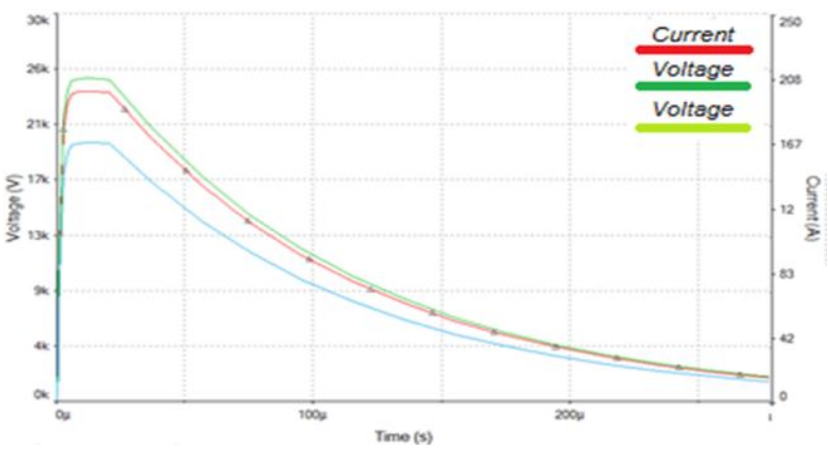

phenomena as observed in the emperical analysis.

Figure 6. NI Multisim Transient Plot From conventional Model

Figure 7. NI Multisim Transient Plot From Improved Model
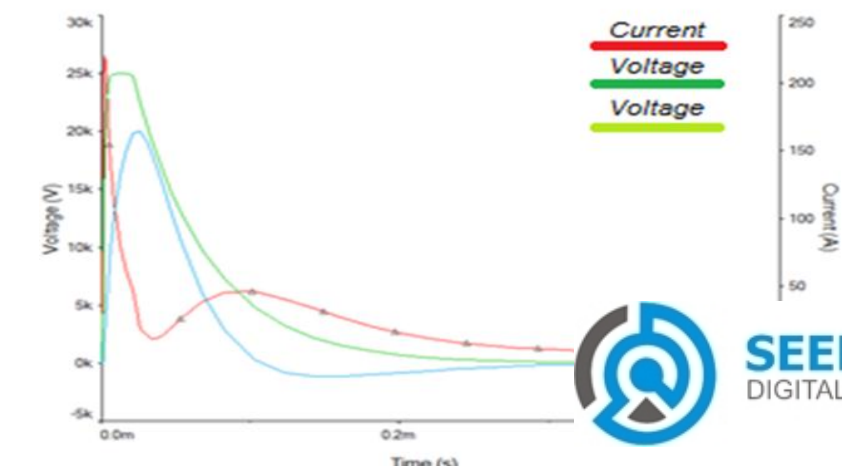


\section{Conclusion}

In this article, we extensively studied and reported a model for the soil under impulse discharge current using, circuit and numerical models to validate empirical results from previous experiments until the results obtained are seen to be consistent with one another.

The soil properties where evaluated and it was reordered that the variation in soil from one place to another is majorly related to the available moisture, $\mathrm{pH}$ and micronutrient. These factors altogether have a strong influence on the conductivity and stability of the soil at any locations. This properties were also observe to be a function of time as it isn't constant. The resistance and resistivity measured were also seen to vary not only with depth but across suggesting soil are not just having a multilayer or stratified layering system.

It was observed that the current distribution is strongly affected by the conductivity of the soil and the distance from localized discharge point. The presence of a secondary breakdown and soil ionization were also observed validating the presence of micro voids and strengthening the stratified layer assumptions as energization of current became evident. An equivalent circuit model was developed to solve the complex-impedance problem of soil resistivity/ conductivity as a function of reactance and resistance taken into account the secondary breakdowns that might occur at critical electric potential causing soil ionization and further lowering the impedance. The optimum soil model was found to be comprising of a capacitor in parallel to a resistor in series with an inductor $(\mathrm{C} \| \mathrm{R}+\mathrm{L})$. The addition of an inductive element into the overall model whose result is shown in Fig. 7 is concluded to be responsible for modelling the second peak after secondary soil ionization. The developed equations would hold for both linear and nonlinear time varying complex soil impedance respectively.

In conclusion, the current distribution in soil surface and that traversing through the soil is strongly related to the conductivity of the soil which happens not to be a constant value nor a variable with just one coefficient rather a combination of variables best modelled as a complex impedance.

\section{Acknowledgment}

This research has been supported by Malaysian Ministry of Higher Education Research Grant, RAGS15-063-0126.

\section{References}

[1] M. Mokhtari, Z. Abdul-Malek, Z. Salam, An Improved Circuit-Based Model of a Grounding Electrode by Considering the Current Rate of Rise and Soil Ionization Factors, IEEE Trans. Power Deliv. 30 (2015) 211-219. doi:10.1109/TPWRD.2014.2347283.

[2] F.E. Fajingbesi, N.S. Midi, E.M.A. Elsheikh, S.H. Yusoff, High Voltage Discharge Profile on Soil Breakdown Using Impulse Discharge, in: IOP Conf. Ser. Mater. Sci. Eng., 2017. doi:10.1088/1757-899X/210/1/012040.

[3] F.E. Fajingbesi, N.S. Midi, E.M.A. Elsheikh, S.H. Yusoff, Discharge current distribution in stratified soil under impulse discharge, in: IOP Conf. Ser. Mater. Sci. Eng., 2017. doi:10.1088/1757899X/210/1/012057.

[4] S. Liu, W. Sima, T. Yuan, D. Luo, Y. Bai, M. Yang, Visualization of the inner discharge of soil under impulse current and calculation of the ionization parameters, in: 2016 33rd Int. Conf. Light. Prot. ICLP 2016, 2016. doi:10.1109/ICLP.2016.7791480.

[5] L. Cheng, Z. Kong, S. Wang, W. Qi, Z. Li, L. Pu, J. Wu, J. Li, Study on spark discharge characters of soil under lightning impulse, in: Proc. 2016 IEEE Int. Conf. Dielectr. ICD 2016, 2016: pp. 1134-1138. doi:10.1109/ICD.2016.7547817

[6] V.L. Coelho, L.F. Venturini, H. a D. Almaguer, R. a Coelho, A. Piantini, Wallace C. Boaventura, J.O.S. Paulino, P.L. Nosaki, Soil Resistivity Behavior under High Current Density Values Using the Energy Balance Method, Int. Symp. Light. Prot. (XIII SIPDA). (2015) 207-214. doi:10.1109/SIPDA.2015.7339331.

About Author (s):

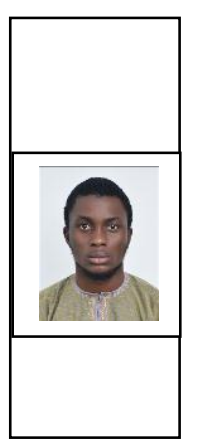

Fawwaz Eniola Fajingbesi

B.Sc. Physics with electronic, Fountain University Osogbo Nigerian and MSc. Electronics Engineering, PhD Candidate at the Electrical and Computer Engineering Department, International Islamic University Malaysia. He is on study leave from Fountain University Osogbo, Nigeria.

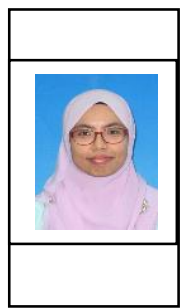

Nur Shahida Midi

PhD Engineering, Tokai University, Japan She is an Assistant Professor at the Electrical and Computer Engineering Department, International Islamic University Malaysia.

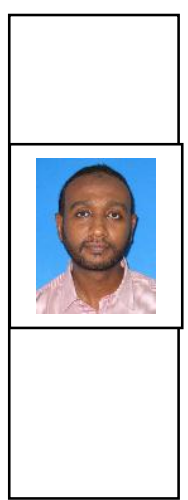

Elsheikh Mohamed Ahmed Elsheikh

Had is bachelor's degree in Telecommunication, University of Khartoum; M.Sc Radio Systems Engineering, University of Hull; $P h D$ Telecommunication/Information Theory, University College London.

$\mathrm{He}$ is an Assistant Professor at the Electrical and Computer Engineering Department, International Islamic University Malaysia

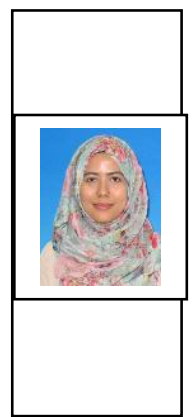

Siti Hajar Yusoff

Had her bachelor's degree in Engineering (Electrical/Electronic), University of Nottingham, PhD Engineering (Electrical/Electronic), University of Nottingham. She is an Assistant Professor at the Electrical and Computer Engineering Department, International Islamic University Malaysia. 